\title{
Der Arbeitsrechtsrechtsdiskurs und die Rechtsempirie - Ein schwieriges Verhältnis
}

Obwohl sich die Situation am Arbeitsmarkt verbessert hat, dauert die Debatte um das Problem der Massenarbeitslosigkeit an. Welche Lösungsansätze dafür in der Rechtswissenschaft diskutiert werden, wird am Kündigungsschutz gezeigt. Dies Beispiel macht anschaulich, wie resistent sich die Rechtswissenschaft gegenüber empirischen Forschungsbefunden zeigt. Allerdings sind bei der nach wie vor starken Gruppe der Kritiker des geltenden Kündigungsschutzes Akzentverschiebungen in der Argumentation erkennbar, jedoch kein Abrücken von ihrer oft (fundamtental-)kritischen „Wertehaltung“. Gleichwohl ist die empirische Forschung nicht folgenlos. Der Beitrag versucht, sich diesem komplexen Thema zu nähern und Anstöße für Forschung und Rechtspolitik zu geben. ${ }^{1}$

\section{Hauptlinien der arbeits- rechtlichen Diskussion zum Kündigungsschutz}

In der arbeitsrechtswissenschaftlichen Debatte steht der Kündigungsschutz seit Langem im Brennpunkt der Kontroversen. Dabei geht es nicht um Auseinandersetzungen über Auslegungsfragen und Einzelheiten der kündigungsrechtlichen Regelungen. Vielmehr gibt es - und das ist die entscheidende Besonderheit, auch im Vergleich zu anderen Arbeitsrechtmaterien, - eine sehr fundamentale Kritik, die sich auf das Gesetz selbst richtet bzw. die aus seinen vermeintlichen Wirkungen auf dem Arbeitsmarkt resultiert.

Zum einen wird behauptet, das Arbeitsrecht sei insgesamt zu starr. An vorderer Stelle für Fehlsteuerungen stehe insoweit der (materiell) überzogene Kündigungsschutz. Seine Rigiditäten würden nicht nur die Arbeitskosten in die Höhe treiben, sondern auch die notwendigen Anpassungen im Strukturwandel behindern (Möschel 2006, S. 113). Dieses Argument zielt darauf, den Kündigungsschutz als Hemmnis für die Flexibilität des Arbeitsmarktes zu markieren.

Zum Zweiten und damit zusammenhängend wird vorgetragen, dass die negative Situation des Arbeitsmarktes auf Überregulierungen zurückzuführen sei. $\mathrm{Zu}$ den Ansatzpunkten für eine Modernisierung des Arbeitsrechts, die eine dringend notwendige Vereinfachung und Beschleunigung der Abläufe verbessern könnte, gehöre vorrangig das Kündigungsschutzrecht (z. B. Löwisch 2005a, 2005b, S. I; Bauer
2005, S. 1046ff.; dagegen z. B. Huber 2005, S. 1340ff.). Die Zuschreibung, überkomplex und bürokratisch zu sein, macht den Kern dieses Kritikpunktes am Kündigungsschutz aus.

Dazu komme drittens, dass nicht nur die Regeln selbst in unerträglicher Weise Rechtsunsicherheit ausstrahlten (Bauer, a.a.O.). Vielmehr habe die (höchstrichterliche) Rechtsprechung die gesetzlichen Generalklauseln, insbesondere „die soziale Rechtfertigung" nach $\$ 1$ KSchG, im Wege einer unbegrenzten Auslegung zulasten der Arbeitgeberseite grundlegend umgedeutet. Hierzu trage insbesondere bei, dass die Kündigung nach der Rechtsprechung lediglich als „ultima ratio“ in Betracht komme und zudem eine "Prognose“ über die zukünftige Entwicklung verlangt werde. Dies alles führe zu unangemessenen Ergebnissen, was zur Folge habe, dass kleinere und mittlere Unternehmen davon abgeschreckt würden, Neueinstellungen vorzunehmen.

Diese Ansicht, die eine Fundamentalkritik an der Rechtsprechung widerspiegelt, wird im Arbeitsrecht seit vielen Jahren dezidiert von prominenter wissenschaftlicher Seite mit Breitenwirkung und meinungsbildend für das politische Umfeld vertreten (Rüthers 2006, S. 1640ff.; SVR 2003/04, Rn. 677ff., 681f.). Zur Illustration weist man auf einige Beispiele aus der Rechtsprechung hin, so auf den Fall eines U-Bahn-Fahrers der Berliner Verkehrsbetriebe, der am Steuer seines Pkw volltrunken angetroffen wurde, dessen verhaltensbedingte Kündigung dennoch durch alle drei Instanzen nicht akzeptiert worden war (BAG 4.6.1997, Arbeitsrechtliche Praxis Nr. 137 zu $₫ 626$ BGB).

\section{Arbeitsrecht und Industrial Relations-Forschung}

Nun gibt es kaum ein arbeitsrechtliches Gebiet, das besser erforscht ist als der Kündigungsschutz. Wegweisend war zunächst die „Max-Planck-Studie“ aus dem Jahr 1981 mit einer umfassenden Befragung der von Kündigungen Betroffenen und Beteiligten an Kündigungsschutzverfahren sowie einer Auswertung von Kündigungsschutzakten (Falke et al. 1981). Auch das Projekt „Regulierung des Arbeitsmarktes“ („REGAM“) des WSI in der Hans-BöcklerStiftung hat 2001 und 2003 repräsentative empirische Studien zum Kündigungsschutz durchgeführt (Pfarr et al. 2005). Fast zeitgleich untersuchte eine Forschungsarbeit der Universität Halle-Wittenberg 2003 empirisch die Kündigungspraxis und den Kündigungsschutz im Arbeitsverhältnis („KÜPRAX“, Höland et al. 2007).

Alle diese Studien stellten fest, dass eine negative Einwirkung des Kündigungsschutzes auf das Einstellungs- und Beendi-

\footnotetext{
1 Die Überlegungen gehen zum großen Teil auf das Projekt "Arbeitsrecht in der betrieblichen Praxis (Ariba)“" zurück, das der Verfasser gemeinsam mit Prof. Florian Schramm durchgeführt hat.
}

Ulrich Zachert, Prof. Dr., Universität Hamburg, Department für Wirtschaft und Politik. Arbeitsschwerpunkte: Arbeitsrecht, Europäisches Recht, Rechtsvergleichung. e-mail:ulrichzachert@aol.com 
gungsverhalten der Arbeitgeber empirisch nicht $\mathrm{zu}$ belegen ist, dass Beendigungen weitgehend konfliktfrei verlaufen und die Arbeitsgerichtsbarkeit, die selten angerufen wird, schnell und durchaus abgewogen entscheidet. Auch die Kosten des Kündigungsschutzes, insbesondere Abfindungen, sind geringer und fallen seltener an als in der öffentlichen Debatte behauptet.

Weitere, eher ökonomisch ausgerichtete Untersuchungen bestätigen ganz überwiegend, dass der bestehende Kündigungsschutz keine signifikanten Auswirkungen auf den Arbeitsmarkt hat (OECD 1999, S. 51; OECD 2004, S. 260; Bauer et al. 2004). Allerdings wird hier die These formuliert, dass ein Zusammenhang zwischen Kündigungsschutzniveau und einer hohen Arbeitslosigkeit bestimmter Gruppen, besonders die jüngerer Arbeitnehmer, bestehen könnte.

Nur vereinzelt wird aus empirischen Umfragen abgeleitet, der geltende Kündigungsschutz verhindere die Schaffung neuer Arbeitsplätze (Janßen 2004). Dabei handelt es sich um Meinungsumfragen (kritisch zur Methode: Schramm/Zachert 2006).

\section{Rezeption der Empirie in der arbeitsrechtlichen Diskussion}

\subsection{AKZENTVERSCHIEBUNG: STIMMUNGEN, GEFÜHLE, ÄNGSTE}

Die empirischen Ergebnisse geben in ihrer übergroßen Mehrheit keinen Anlass für Erwartungen oder Hoffnungen, ein „Drehen an der Stellschraube des Kündigungsschutzes" würde signifikante Ergebnisse auf dem Arbeitsmarkt zur Folge haben (Neubäumer 2005, S. 25, 32f.).

Die Auswirkungen dieser Forschungsbefunde auf die rechtswissenschaftliche Debatte über Veränderungsnotwendigkeiten erscheinen dagegen eher gering und sogar ambivalent. Wenig überrascht, dass aus Teilaussagen der Empirie unterschiedliche Schlussfolgerungen gezogen werden. ${ }^{2}$ Bemerkenswert erscheint dagegen, dass nach wie vor Vorschläge gemacht werden, die eine radikale Umgestaltung des geltenden Kündigungsschutzes befürworten (Bauer 2005; Möschel 2006). Allerdings ist bei den (Fundamental-)Kritikern des geltenden
Kündigungsschutzes eine Akzentverschiebung in der Begründung ihrer Vorschläge zu beobachten. Insbesondere Meinungsführer in dieser Kontroverse stellen die harten Fakten der mehrheitlich vorliegenden Ergebnisse an sich nicht (mehr) infrage. Stattdessen wird nunmehr schwerpunktmäßig die vermeintliche Undurchschaubarkeit des geltenden Kündigungsschutzes kritisiert. Damit wird vorrangig auf Stimmungen, Gefühle und Ängste von Arbeitgebern abgehoben. Unter anderem verweist man darauf, dass Inhaber von Kleinstunternehmen, die nicht unter das Kündigungsschutzgesetz fallen, den Kündigungsschutz gleichwohl als Last empfinden und zugleich mehrheitlich angeben, ohne ihn wären sie eher zu Neueinstellungen bereit (Möschel 2006, S. 113; Rüthers 2006, S. 1640f.).

Psychologische Faktoren wie Rechtsgefühl, Rechtsbewusstsein und Rechtsakzeptanz lassen sich in der Tat mit quantitativer Empirie nur schwer einfangen. Mit solchen Argumenten ist es jedoch am ehesten möglich, die eigene Position gegen einschlägige quantitative Ergebnisse der Empirie weitgehend zu immunisieren oder Letztere jedenfalls in starkem Umfang zu entwerten. Folgerichtig wird neuestens behauptet, dass sich diese affektiven Aspekte der empirischen Forschung grundsätzlich entziehen würden (Mohr 2006, S. 547, 556).

\subsection{THEMATISCHE KONTINUITÄTEN}

Thematisch kreist die Kontroverse um „Sinn und Widersinn des Kündigungsschutzes" nach wie vor zum einen um die Frage, ob das Kündigungsschutzgesetz eine prohibitive Wirkung habe, also ob dessen negative Wahrnehmung und Einschätzung die Unternehmer und Personalverantwortlichen davon abhalte, Arbeitnehmer einzustellen, oder ob sie deshalb gegebenenfalls auf andere personalpolitische Instrumente, wie z. B. Befristung, Leiharbeit oder Überstunden, ausweichen würden. Zum anderen wird eine vermutete präventive Wirkung debattiert. Strittig ist hierbei die Frage, ob das Gesetz Entlassungen verhindere, weil sie nur um den Preis von zeitlichen Verzögerungen oder ökonomisch unvertretbar hohen Abfindungen zu realisieren seien (differenzierende Analyse: Bayreuther 2006, S. 417, 418). In Anlehnung an eine solche "Präventionswirkung“ wird auch behauptet, die relativ geringe Zahl von Kündigungsschutzklagen sei dadurch zu erklären, dass die Gesetze zu streng sei- en und die Arbeitgeber alles tun würden, um Prozesse zu vermeiden (Rieble nach Viering 2005).

Dies ist nichts anderes als in verändertem Gewande die Fortschreibung der alten Hypothese, das Arbeitsrecht würde die betriebliche Wirklichkeit stark und im Hinblick auf die arbeitsmarktpolitischen Effekte negativ beeinflussen. In eine etwas andere Richtung zielt eine Position im Rahmen einer neueren arbeitsrechtlichen Diskussion über "gefühltes und gelebtes Arbeitsrecht“. Sie läuft darauf hinaus, wegen der Undurchschaubarkeit des Arbeitrechts sei die Tendenz erkennbar, sich von ihm zu verabschieden und ohne Rücksicht auf das normierte Recht ein eigenes, realitätsgerechtes Arbeitsrecht zu praktizieren (Kania 2004, S. 1 und 2005, S. 596ff.; dagegen Stein 2006, S. $110 \mathrm{ff}$.).

\subsection{FEHLEINSCHÄTZUNGEN DER WIRKUNG VON ARBEITSRECHT}

An dieser Stelle kann es nicht darum gehen, die Ergebnisse einer kombinierten, qualitativen und quantitativen Befragung von Personalverantwortlichen über ihre Wahrnehmung und Einstellung zum Arbeitsrecht, die diesen Fragen nachgeht (Schramm/ Zachert et al. 2007), im Einzelnen wiederzugeben. Sie laufen stark verkürzt darauf hinaus, dass die Wirkung arbeitsrechtlicher Normen im rechtlichen Diskurs teils über-, zum Teil jedoch unterschätzt wird: Man überschätzt in der Tendenz den Stellenwert des Arbeitsrechts als handlungsleitenden Faktor für Entscheidungen von Personalverantwortlichen, die ganz vorrangig nicht arbeitsrechtlichen, sondern ökonomischen Motiven folgen. Demgegenüber wird seine Präsenz in den Unternehmen in der juristischen Diskussion tendenziell unterschätzt. Die Beobachtung, es gebe ein „gefühltes Arbeitsrecht", das nicht immer mit dem „geschriebenen Recht" übereinstimme, trifft durchaus zu. Jedoch wird das normierte Arbeitsrecht in all seiner Differenziertheit überwiegend „loyal“ angewandt und umgesetzt. Von ,einer Flucht aus dem Arbeitsrecht" kann deshalb keine Rede sein (Tornau 2007, S. 58).

\footnotetext{
2 So schlägt z. B. Preis $(2003$, S. 65,72$)$ eine Ersetzung der Sozialauswahl durch Abfindungslösungen vor; Wolter $(2003$, S. 1068,1071$)$ empfiehlt den vorsichtigen Ausbau der Weiterbeschäftigung.
} 
Nachfolgend sollen zunächst die Faktoren benannt und kurz analysiert werden, die für die Wirkung des Arbeitsrechts eine Rolle spielen. Auf diese Weise erscheint es möglich, Anhaltspunkte darüber zu erhalten, wie es zu bestimmten Einschätzungen von Arbeitsrechts-Praktikern kommt (Abschnitt 4). Auf dieser Grundlage lassen sich dann Antworten gewinnen, inwieweit für empirische Forschungen die Aussicht besteht, von verschiedenen "Zielgruppen“ und in unterschiedlichen Verwendungszusammenhängen zur Kenntnis genommen zu werden (Abschnitt 5).

\section{Einflussfaktoren auf Rechtsgefühl und -verhalten}

\subsection{ARBEITSRECHTLICHER SACH- VERSTAND: VORAUSSETZUNGEN UND INFORMATIONSQUELLEN}

Versucht man die Elemente, die in der rechtssoziologischen Diskussion als für die Rechtsumsetzung relevant genannt werden (Röhl 1987, S. 252ff.; Rottleuthner 1987, S. 57; Raiser 1999, S. 258f.), im Hinblick auf Personalverantwortliche und ihre Arbeit im Unternehmen zu konkretisieren, so erhält man ein facettenreiches Bild.

Zunächst stellt sich die Frage, welcher arbeitsrechtliche Sachverstand zur Verfügung steht und welches die Informationsquellen sind, die die Rechtseinstellungen von Personalverantwortlichen und ihr Verhalten prägen. $\mathrm{Ob}$ arbeitsrechtliche Kompetenz im Unternehmen (z. B. in der Personalabteilung) vorhanden ist oder ob sie extern eingeholt werden muss und an wen man sich wendet - dies alles ist bereits eine entscheidende Weichenstellung für das Verhältnis zum und den Umgang mit Recht.

Untersuchungen bestätigen die nahe liegende Hypothese, dass es insoweit signifikante Unterschiede je nach Unternehmensgröße gibt. Interne arbeitsrechtliche Ressourcen finden sich eher in größeren als in Klein- und Mittelbetrieben (Alewell/ Koller 2002, S. 990ff.; Pfarr et al. 2005, S. 13ff.). Wenn die Beratung extern erfolgt, so kommen u.a. (Fach-)Anwälte, Verbände, aber auch Steuerberater in Betracht. Systematische Kenntnisse lassen sich aus Fachzeitschriften, Fachliteratur und Fortbildung in Fachseminaren erwerben.

\subsection{ALLGEMEINE MEDIEN UND FACHDEBATTE}

Es gibt Anhaltspunkte dafür, dass die in den Medien und in der Fachdebatte veröffentlichte Meinung von nicht zu unterschätzendem Gewicht für das Rechtsverständnis der Rechtspraktiker ist (Castendyk 1994; Hensche 2006, Speth 2004, 2006). In wichtigen Konfliktfeldern steht beides häufig in einer Wechselwirkung. Im Hinblick auf den Kündigungsschutz wurde die Position des Sachverständigenrates maßgeblich durch den Arbeitsrechtler Rüthers geprägt, der hierzu seit Langem eine dezidiert kritische Position vertritt (SVR 2003, S. VII. und Rn. 677ff., 681f.; SVR 2005, Rn. 316, allerdings mit Differenzierungen). Selbst Fehlinterpretationen von Rechtsnormen und ihren Wirkungen prägen Einstellungen zum Recht, allemal, wenn sie von prominenten Wissenschaftlern oder Verbandsvertretern vorgetragen werden. Verwiesen sei auf die Verwechslung von betrieblicher und Unternehmensmitbestimmung (so der Sachverständige W. Franz). Ferner wurde die Behauptung öffentlich formuliert, eine sachgrundlose Befristung nach $\$ 14$ Abs. 2 TzBfG dürfe nur einmal abgeschlossen werden (so „Kronberger Kreis"), oder das Betriebsverfassungsgesetz verbiete kollektive Vereinbarungen über Lohn und Arbeitszeit (so Rogowski als ehemaliger Präsident des BDI). Es ist zu vermuten, dass derart fehlerhafte Auslegungen auch die praktische Anwendung des Arbeitsrechts in den Betrieben (negativ) beeinflussen.

\subsection{SYSTEMATISCHER ODER PUNKTUELLER UMGANG MIT DEM ARBEITSRECHT}

Ein weiterer Gesichtspunkt, der mit dem Vorstehenden zusammenhängt, betrifft den Umgang mit dem Arbeitsrecht im Unternehmen selbst. So wird relevant sein, ob dieser systematisch oder lediglich punktuell (ad hoc) erfolgt. Auch sprechen Hinweise dafür, dass Einschätzungen und Anwendungen des Arbeitsrechts davon beeinflusst werden, $o b$ eine regelmäßige Auseinandersetzung mit Neuerungen stattfindet oder ob ein (eher) reaktives Verhalten vorliegt, sodass man erst tätig wird, „wenn das Kind in den Brunnen gefallen ist", d.h. wenn in einem Konflikt negative Erfahrungen gemacht wurden (Hübner 2005, S. 464ff.).

Hier ist es wiederum notwendig, zwischen Groß-, Mittel- und Kleinbetrieben zu differenzieren. Es gibt Anhaltspunkte dafür, dass die Kultur in Kleinbetrieben eine „besondere soziale Welt" darstellt, die weniger durch das „normative Modell“ geprägt ist, als dies in größeren Unternehmen der Fall ist (vgl. Kotthoff/Reindl 1990; Dibbern-Voß 2005; Hübner 2005, S. 404ff. und 439ff.).

Jedoch stößt man auch unabhängig von Betriebsgrößen auf vielfältige arbeitsrechtliche Wirklichkeiten. Sie differenzieren sich aus nach Branchen und deren spezifischen Kulturen und Traditionen sowie nach Führungsstilen. Insoweit ist auch von Bedeutung, ob es eine „Kultur der Mitbestimmung" gibt und wie sie sich praktisch bewährt (Frick 2005).

\subsection{ANEKDOTISCHE EVIDENZ}

In Befragungen von Personalverantwortlichen wurde auffallend oft deutlich, dass die Befragten angaben, selber keine Probleme im Umgang mit dem Arbeitsrecht zu haben, dies jedoch für andere Branchen oder Unternehmenstypen vermuteten (Schramm/Zachert et al. 2007). Dies belegt den Verdacht, dass die Problemwahrnehmung im Arbeitsrecht in nicht unerheblichem Maße unter dem Einfluss anekdotischer Evidenz zustande kommt.

Das Phänomen der anekdotischen Evidenz ist spätestens seit den REGAM-Studien zu einem Thema der arbeitsrechtspolitischen Diskussion geworden (Pfarr et. al. 2004, S. 193f.). Es bezeichnet eine weitverbreitete Art der „Beweisführung“, deren empirisches Fundament auf Einzelfällen aufbaut. Von solchen Einzelfällen wird unhinterfragt auf das Ganze geschlossen. So scheinen beispielsweise über Medien oder Fachpublikationen verbreitete Meldungen - das gilt insbesondere für exotische Nachrichten - einen nicht unerheblichen Einfluss auf das Zustandekommen von Wahrnehmungen des Arbeitsrechts zu haben, die dann quasi als "Paradebeispiele” eigene Überzeugungen prägen können. Es besteht durchaus die Gefahr, dass sie nicht ohne Einfluss auf die Wahrnehmungen von betrieblichen Rechtspraktikern bleiben, gerade vor dem Hintergrund begrenzter Informationen, aus gutem Glauben oder weil sie Vereinfachung bieten. In gewisser Weise helfen solche Alltagsbeobachtungen den Akteuren, nachzuvollziehen, wie sich arbeitsrechtliche Problemlagen darstellen könnten, selbst wenn Vergleichbares weder für die Mehrzahl der Fälle noch für das ei- 
gene Unternehmen zutreffend ist. Auch kann angenommen werden, dass gerade diejenigen Betriebe, in denen es Probleme gibt, stärker in die öffentliche Wahrnehmung treten als eine mögliche Mehrheit, für die das Arbeitsrecht unproblematisch zu handhaben ist.

Insgesamt beeinflussen jedoch entsprechende Anekdoten und Meldungen in erster Linie die Diskussion der Beteiligten, sie bestimmen dagegen nicht unbedingt das tatsächliche Verhalten der Akteure in ihrer betrieblichen Praxis (Schramm/Zachert et al. 2007 mit weiteren Einzelheiten).

\section{Empirie für wen und weshalb?}

Die dargestellten Erfahrungen mit dem Umgang der Arbeitsrechtswissenschaft mit empirischen Ergebnissen zum Kündigungsschutz sprechen zunächst nicht unbedingt dafür, dass weitere empirische Forschungen in angemessener Form zur Kenntnis genommen werden. Gleichwohl ist der Ansicht zuzustimmen, dass in den Anstrengungen, empirische Forschung zu betreiben, nicht nachgelassen werden sollte. Der Anspruch muss aufrecht erhalten werden, empirisch möglichst verlässliche Daten zu generieren, die die (fach-)öffentliche Debatte immer wieder herausfordern und von ihr - zumindest nicht auseinandersetzungslos - übergangen werden können (Sadowski 2004, S. 231, 235). Insofern bleibt die Rechtswissenschaft selbst ein wichtiger Adressat für die Ergebnisse der Rechtsempirie.

Gleiches gilt mit Blick auf die betrieblichen Akteure - trotz aller Schwierigkeiten, Forschungsergebnisse in die betriebliche Praxis hinein zu kommunizieren. Die Analyse rechtlicher Wirkungen und arbeitsweltlicher Realität ist unverzichtbar, damit unerwünschte oder unerwartete Nebenfolgen in das Blickfeld der Praxis gelangen, vermieden oder korrigiert werden können. Zwei Beispiele für Fälle, in denen die empirische Rechtsforschung unvermutete Wir- kungszusammenhänge offengelegt hat: Es konnte gezeigt werden, dass der Kündigungsschutz nicht nur in Form seines Normgehalts, sondern auch im Hinblick auf Faktoren wie „Vertrauen“ wirkt. Großflächige oder als ungerechtfertigt erscheinende Kündigungen führen zu Vertrauensverlusten in der verbleibenden Belegschaft und Imageverlusten für das Unternehmen (Stahlmann et al. 2004). Unsichere Arbeitsplätze können ferner auf andere gesellschaftliche Felder, etwa auf die Familienpolitik, ausstrahlen, indem z. B. die demografische Entwicklung negativ beeinflusst wird (einerseits Mundorf 2006, S. 107; Zachert 2006; andererseits Rieble 2006).

Was den Gesetzgeber betrifft, so ist die Aussicht besonders groß, dass er bei Vorbereitung wichtiger parlamentarischer Initiativen, bei Anhörungen und ähnlichem auf Expertenwissen und Analysen über die tatsächlichen Voraussetzungen und Wirkungen von Normen zurückgreift. Dass bei allen Veränderungen, die der Kündigungsschutz im vergangenen Jahrzehnt erfahren hat, auf der Gesetzgebungsebene empirische Untersuchungen Berücksichtigung finden, die dessen Wirkung auf den Arbeitsmarkt differenziert und kritisch sehen, zeigen Beispiele aus jüngster Vergangenheit (Deutscher Bundestag 2004, S. 5). Allerdings verfügt der Gesetzgeber über eine Einschätzungsprärogative im Sinne eines weiten rechtspolitischen und prognostischen Freiraums. Gleichwohl ist er generell gehalten, sorgfältige Ermittlungen und verlässliche Prognosen anzustellen (Blum 2004; European Commission 2005). Das gilt namentlich bei möglichen Grundrechtsgefährdungen und hat $z$. B. eine Bedeutung für den (begrenzten) Bestandsschutz des Arbeitsverhältnisses (vgl. BVerfG 27.1.1998, BVerfGE 97, S. 169, 177ff.). Inwieweit dies im Sinne einer Plausibilitätskontrolle rechtlich überprüft werden kann, ist freilich umstritten (vgl. Dieterich 2006, GG Einl. Rn. 42ff.; Ullmann 2006).

Und schließlich hat die Rechtsprechung die Folgen ihrer Entscheidungen zu ermitteln, zu prognostizieren und abzuschätzen.
Dies betrifft insbesondere Wertungen im Rahmen gesetzlicher Generalklauseln. Eine solche Rechtsfolgenabschätzung ist jedenfalls als Appell zur selbstkritischen Reflexion außerrechtlicher Gegebenheiten und Wertungsfragen grundsätzlich anerkannt (Dieterich 2006, GG Einl. Rn. 44) und wird praktiziert (Beispiel zum Kündigungsschutz: BVerfG 27.1.1998, BVerGE 97, S. 169,177ff.). Die Position eines „empirienihilistischen Standpunktes" blieb bislang vereinzelt und hat sich nicht durchgesetzt (zur Diskussion: Karpen 2006; Däubler 2006). Die Bedeutung der Folgenabschätzung ist, namentlich für das Arbeitsrecht, nicht zu unterschätzen, denn sie trägt maßgeblich zur Friedensfunktion (Rechtsakzeptanz) der jeweiligen Entscheidungen bei.

\section{Fazit}

Im Ergebnis kann festgehalten werden: Die vielfach bereits vorliegende empirische Forschung stellt für die Rechtsanwender eine ständige Herausforderung dar. Zugleich gibt es viele gute Gründe, die Rechtstatsachenforschung fortzuführen, zu intensivieren und ihre Ergebnisse für die verschiedenen Zielgruppen sachgerecht aufzuarbeiten (Röhl 2005, S. 1, 8ff., 24ff.). Gesetzgeber, Richter und vor allem die Praxis sind auf sie angewiesen (Gamillscheg 2006, S. 165; Raiser 2007). All dies verweist auf das klassische Thema, dass Werthaltung und Wertewandel durch die komplexe Wechselwirkung von „Sein und Sollen“ nachhaltig beeinflusst werden (Zachert 2004, S. 1ff.).

Die Schwierigkeiten, die sich im Verhältnis zwischen dem rechtswissenschaftlichen Diskurs und der Rechtsempirie zeigen, sind überwindbar, gerade weil die Erfassung der Realität eine solide Aufarbeitung der jeweiligen Fakten verlangt. Dies aber setzt voraus, dass die Rechtsempirie ein eigenes überzeugendes Deutungsmuster entwickelt und in der Lage ist, dieses auch kommunikativ durchzusetzen (Beardwell 1996, S. 8f.). 
Alewell, D./Koller, P. (2002): Arbeitsrechtliche Ressourcen und Einschätzungen in Personalabteilungen deutscher Unternehmen, in: Betriebs-Berater 19, S. 990-992

Bauer, J.-H. (2005): Arbeitsrechtlicher Wunschkatalog für mehr Beschäftigung, in: Neue Zeitschrift für Arbeitsrecht 18, S. 1046-1051

Bauer, K./Bender, S./Bonin, H. (2004): Betriebe reagieren kaum auf Änderungen beim Kündigungsschutz, in: IAB Kurzbericht 15, S. 1-4

Bayreuther, F. (2006): Thesen zur Reform des Kündigungsschutzes, in: Neue Zeitschrift für Arbeitsrecht 8, S. 417-421

Beardwell, I. (1996): How do we know how it really is? An analysis of the new industrial relations, in: Beardwell, I. (Ed.), Contemporary Industrial Relations, Oxford

Blum, P. (2004): Wege zu besserer Gesetzgebung - sachverständiger Beratung, Begründung, Folgeabschätzung und Wirkungskontrolle, in: Gutachten I zum 65. Deutschen Juristentag, Bonn

Castendyk, O. (1994): Rechtliche Begründungen in der Öffentlichkeit Ein Beitrag zur Rechtkommunikation in den Massenmedien, Opladen Däubler, W. (2006): Folgenabschätzung im Arbeitsrecht - zwischen Schutzprinzip und Effizienzgedanken, hektografiertes Manuskript,

S. 1-17

Deutscher Bundestag (2004): Antwort der Bundesregierung auf die Kleine Anfrage der Abgeordneten G. Weiß etc., BT-Drucks. 15/2932

Dibbern-Voß, S. (2005): Arbeitsrecht, Personalmanagement und Betriebsgrößen, in: Schramm, F./Zachert, U.(Hrsg.): Arbeitsrecht - Personalpolitik - Wirklichkeit, Baden-Baden, S. 404-438

Dieterich, T.(2006): in: Erfurter Kommentar zum Arbeitsrecht, 6. Aufl., München

European Commission (2005): Impact Assessment Guidelines 15.6.2005 in: SEC (2005) 791 = http://ec.europa.eu/governance/impact/index_ en.htm.

Falke, J./Höland, A./Rhode, B./Zimmermann, G. (1981): Kündigungspraxis und Kündigungsschutz in der Bundesrepublik Deutschland, Bd. I und II, Bonn

Frick, B. (2005): Rechtsökonomische Kontrolle und Performance der mitbestimmten Unternehmung. Rechtsökonomische Überlegungen und empirische Befunde, in: Kölner Zeitschrift für Soziologie und Sozialpsychologie 45, S. 418-440

Gamillscheg, F. (2006): Ausgewählte Schriften zu Arbeitsrecht und Rechtsvergleichung, Baden-Baden

Hanau, P. (2005): Arbeitsrecht im ökonomischen Zerr-Spiegel, in: Neue Juristische Wochenschrift 17, S. 1173-1174

Hensche, D. (2006): Wissenschaft, Politik, Ideologie, in: Schäfer, C./ Seifert, H. (Hrsg.): Kein bisschen leise: 60 Jahre WSI, Hamburg, S. 13-31 Höland, A./Kahl, U./Zeibig, N. (2007): Kündigungspraxis und Kündigungsschutz im Arbeitsverhältnis, Baden-Baden

Huber, G. (2005): Beschäftigungsfördernder Wunschkatalog, in: Neue Zeitschrift für Arbeitsrecht 23, S. 1340-1342

Hübner, S. (2005): Die Umsetzung von Arbeitsrecht in die betriebliche Wirklichkeit, in: Schramm, F./Zachert, U. (Hrsg.): Arbeitsrecht-Personalpolitik und Wirklichkeit, Baden-Baden, S. 439-476

Janßen, P. (2004): Arbeitsrecht und unternehmerische Einstellungsbereitschaft, in: iw-trends (Hrsg.): Initiative Neue soziale Marktwirtschaft, S. $16-25$

Kania, Th. (2004): Angebliches Arbeitsrecht, in: Betriebs-Berater 42, S. I
Kania, Th. (2005): Geschriebenes und geltendes Arbeitsrecht, in: WSIMitteilungen 10, S. 596-598

Karpen, U. (2006): Folgenabschätzung für Gesetze und Richterrecht hektografiertes Manuskript, S. 1-25

Kotthoff, H./Reindl, J. (1990): Die soziale Welt kleiner Betriebe - Wirtschaften, arbeiten und leben im mittelständischen Industriebetrieb, Göttingen

Löwisch, M. (2005a): BB-Forum: Maßnahmen zur Vereinfachung und Beschleunigung im Arbeitsrecht, in: Betriebs-Berater 47, S. 2580-2583

Löwisch, M. (2005b): Vereinfachung und Beschleunigung im Arbeitsrecht - 15 Vorschläge, in: Betriebs-Berater 31, S. I

Mohr, J. (2006): Beschäftigungsförderung durch Kündigungsförderung, in: Zeitschrift für Arbeitsrecht 37, S. 547-593

Möschel, W. (2006): Kündigungsschutz und Beschäftigung - ein Scheinproblem?, in: Juristenzeitung 3, S. 113-118

Mundorf, H. (2006): Nur noch Markt, das ist zu wenig, Hamburg

Neubäumer, R. (2006): Der gesetzliche Kündigungsschutz aus ökonomischer Sicht, in: Sozialer Fortschritt 1, S. 25-34

OECD (1999): Employment Outlook, Paris

OECD (2004): Employment Outlook, Paris

Pfarr, H./Ullmann, K./Bradtke, M./Schneider, J./Kimmig, M./Bothfeld, S.

(2005): Der Kündigungsschutz zwischen Wahrnehmung und Wirklichkeit, München und Mehring

Preis, U. (2003): Reform des Bestandsschutzrechts im Arbeitsverhältnis, in: Recht der Arbeit 2, S. 65-81

Raiser, Th. (1999): Das lebende Recht, Rechtssoziologie in Deutschland, 3. Aufl., Baden-Baden

Raiser, Th. (2007): Krise der Rechtssoziologie in Deutschland, in: Neue Juristische Wochenschrift 29, Editorial

Rieble, V. (2006): Antidiskriminierung und Familien, in: Betriebs-Berater 30, S. 1

Röhl, K. F. (1987): Rechtssoziologie, Köln u.a.

Röhl, K. F. (2005): Recht und Wissenschaft als Thema der Rechtssoziologie, in: Zeitschrift für Rechtssoziologie 5, S. 3-34

Rottleuthner, H. (1987): Einführung in die Rechtssoziologie, Darmstadt Rüthers, B. (2006): Der geltende Kündigungsschutz - Beschäftigungsbremse oder Scheinproblem?, in: Neue Juristische Wochenschrift 23,

S. 1640-1642

Sachverständigenrat zur Begutachtung der gesamtwirtschaftlichen Entwicklung (SVR) (2003): Jahresgutachten 2003/04. Staatsfinanzen konsolidieren - Steuersystem reformieren, November

Sachverständigenrat zur Begutachtung der gesamtwirtschaftlichen Entwicklung (SVR) (2005): Jahresgutachten 2005/2006, Die Chance nutzen - Reformen mutig voranbringen, November Sadowski, D. (2004): Betrieb-Arbeitsmarkt-Interessenvertretung: Herausforderungen an ein Erfolgsmodell, in: WSI-Mitteilungen 5, S. 231-236 Schramm, F./Zachert, U. (Hrsg.) (2005): Arbeitsrecht-PersonalpolitikWirklichkeit, Baden-Baden

Schramm, F. /Zachert, U. (2006): Arbeitsrecht: Kaum Einfluss auf neue Jobs, in: Böcklerimpuls 14, S. 3

Schramm, F./Zachert, U. (2007): Arbeitsrecht in der betrieblichen Anwendung (AribA), Endbericht (unveröffentlicht)

Speth, R. (2004): Die politischen Strategien der INSM, Arbeitspapier 96 der Hans-Böckler-Stiftung 
Speth, R. (2006): Die zweite Welle der Wirtschaftskampagnen - Von "Du bist Deutschland“ bis zur "Stiftung Marktwirtschaft" , Arbeitspapier 127 der Hans-Böckler-Stiftung Stahlmann, M./Wendt-Kleinberg, W./Weyrather, I. (2004): Zwischen Engagement und innerer Kündigung - Arbeitsemotionen im Prozess fortschreitenden Personalabbaus, in: Industrielle Beziehungen 4, S. 380-394 Stein, P. (2006): Abschied vom Arbeitsrecht?, in: WSI-Mitteilungen 2, S. 110-113

Tornau, J. (2007): Belastbar und funktional, in: Mitbestimmung 5, S. 58-59
Ullmann, K. (2006): Arbeitsrechtspolitik ohne Tatsachengrundlage, in: Kritische Justiz 1, S. 26-38

Viering, J. (2005): Arbeitsgerichte erschweren Kündigungen kaum, in: Süddeutsche Zeitung vom 15.4 .

Wolter, H. (2003): Reformbedarf beim Kündigungsrecht aus Arbeitnehmersicht, in: Neue Zeitschrift für Arbeitsrecht 19, S. 1068-1076

Zachert, U. (2004): Legitimation arbeitsrechtlicher Regelungen aus historischer und aktueller Sicht, in: Recht der Arbeit 1, S. 1-8

Zachert, U. (2006): Die Deutschen sterben aus - na und?, in: Neue Zeitschrift für Arbeitsrecht 12, S. 1 (Editorial)

\title{
Gleichstellung ohne Vereinbarkeit - Vereinbarkeit ohne Gleichstellung? Zum Verhältnis von Gleichstellungspolitik und Familienpolitik
}

\author{
Veranstaltung des WSI \\ 27./28. September 2007 in Berlin
}

Die Vereinbarkeit von Beruf und Familie ist in den vergangenen Jahren zu einem der wichtigsten sozialpolitischen Themen geworden - für die Europäische Union, deren Europäische Beschäftigungsstrategie auf die Erhöhung der Frauenbeschäftigung ausgerichtet ist, wie auch für die deutsche Bundesregierung, die Frauen als Fachkräfte entdeckt hat und die sinkende Geburtenrate als Bedrohung für den Erhalt der sozialen Sicherungssysteme interpretiert. Ein solcher Handlungsansatz ist also nicht primär gleichstellungspolitisch, sondern vorwiegend ökonomisch motiviert. Wie ist er aus gleichstellungspolitischer Perspektive zu bewerten?

Die Veranstaltung setzt die mit den beiden Vorgängertagungen „Strategien der Gleichstellung von Frauen und Männern in der Privatwirtschaft“ (2005) und „Geschlechterverhältnisse im Betrieb: Gleiche Verhältnisse - gleiche Chancen?“ (2006) begonnene Debatte und wissenschaftliche Analyse fort. Die Tagung 2007 legt ihren Fokus auf das Verhältnis von Familien- und Gleichstellungspolitik und thematisiert flankierend Veränderungen in der Arbeitswelt und in den Haushalten. Zielgruppen der Veranstaltung sind Wissenschaftlerinnen und Wissenschaftler, Politikerinnen und Politiker, Gewerkschafterinnen und Gewerkschafter, Gleichstellungsbeauftragte, Studierende und andere Interessierte.

Es referieren u.a. Karin Gottschall, Karin Jurczyk, Hildegard Nickel, Ulla Björnberg, Helma Lutz und Hildegard Theobald.

Weitere Informationen: www.boeckler.de/veranstaltungen

Anmeldung: Katharina Kruse, Hans-Böckler-Stiftung, e-mail: katharina-kruse@boeckler.de, Tel.: 0211/7778-111 